PACS 77.70.+a; 85.60.-q

\title{
Multifunctional devices based on induced polar states
}

\author{
V.F. Kosorotov, L.V. Shchedrina, L.V. Levash \\ Institute of Physics, NAS of Ukraine, 46, prospect Nauky, 03028 Kyiv, Ukraine \\ E-mail: kosorot@iop.kiev.ua; shched@iop.kiev.ua; levash@iop.kiev.ua
}

\begin{abstract}
Search for new pyroactive effective materials with controlled polarization properties is carried out with the goal of developing the new generation of pyroelectric sensors with extended functional capabilities. Elaborated multifunctional pyroelectric device - watt-joulemeter combines the functions of the meter of radiation power and that of radiation energy. Proposed technology based on a consideration of the electric potential spatial distribution in piezoelectric crystals under the inhomogeneous heating conditions provides the needed requirements for a formation of two measuring channels on the common sensitive element. Electronic treatment of output signals provides a simultaneous measurement of the power and energy of radiation pulses.
\end{abstract}

Keywords: induced pyroactivity, tertiary pyroelectric effect, multifunctional sensors, infrared optoelectronics.

Manuscript received 07.06.05; accepted for publication 25.10.05.

\section{Introduction}

Semiconductor and dielectric materials belonging to ten polar crystallographic classes are important functional media for a variety of applications including pyro- and piezoelectric devices for infrared detection and thermal imaging [1]. Pyroelectric IR sensors based on these traditional pyroactive materials are of a rather limited application due to a strong absorption of radiation flux in the middle IR spectral range, which causes the overheating and is followed by destruction of a sensor sensitive element and as well as severe nonlinear distortions of the output signal. The lack of the necessary transparency of pyroelectrics in the IR spectral range is a fundamental property of the polar crystals caused by the availability of the spontaneous electric moment that is responsible for their pyroactivity.

The following progress in the field of pyroelectric devices is associated with a search for new functional and intelligent pyroactive materials for a modern infrared optoelectronics. Our studies of the last years [13] proved that nonpolar piezoelectrics that do not belong to pyroelectric classes can also reveal the pyroactive properties under nonequilibrium thermal conditions. Occurrence of pyroactivity in nonpolar crystals is caused by the presence of the thermoelastic stresses generated as a result of the crystal inhomogeneous heating. Mechanical stresses, in their turn, induce polarization in the crystal through the piezoelectric effect. This mechanism of polarization is known as a tertiary pyroelectric effect (TPE) [4, 5].
Among physical effects involving the polarization response to inhomogeneous heating, TPE is the most promising for practical applications. The choice of the method for pyroactive structure formation strongly depends on the problem to be solved. Originally the idea of application of materials not belonging to pyroelectric classes as pyroactive structures was put forward in our study [2] with the goal of increasing the limit for measurable densities of energy and power. It is evident that it becomes possible only on the basis of physical effect manifested itself in crystals possessing a high level of transparency at the wavelength of a laser in use. At the same time, there is an added reason for a peculiar interest in unconventional polar materials for infrared optoelectronics - the possibility exists of developing the multifunctional receiving devices on the base of TPE. The proposed watt-joulemeter is acknowledged to be among the most efficient devices available used for a simultaneous measurement of the energy and power of a laser pulse by the common sensitive element. Extra electronic treatment of the output signal is not necessary. In such a manner, the uncooled pyroelectric sensors based on induced pyroactive structures become a foundation for a new branch of the infrared optoelectronics.

\section{Physical principles for creating the induced pyroactive materials}

A distinguishing feature of TPE is that, under inhomogeneous heating conditions, the polar state in the 
crystal is characterized by a rather intricate spatial distribution of the polarization over the whole crystal volume. The possibility of a practical utilization of such a state can be checked only if we know the spatial distribution of the electrical potential in the crystal and are liable to develop a system of signal electrodes corresponding to this distribution. The lack of any proposals on the practical application of TPE was associated with the following problem. An approximate polarization response to spatially inhomogeneous heating the nonpolar piezoelectric is described by the following expression [5]:

$P_{i}=d_{i \lambda} \sigma_{\lambda}(r, t)$

where $\sigma_{\lambda}(r, t), d_{i \lambda}$ are the tensor components of thermoelastic stresses and piezoelectric moduli. It is well known [6] that, in traditional pyroelectric IR sensors based on the primary and secondary pyroelectric effects, the output signal is proportional to the mean value of the polarization in the crystal volume, i.e., the mean value of the thermoelastic stress tensor is found to accord to (1). This value is expressed in terms of a surface integral of external forces acting on the plate surface and, in the case of their lack, it is equal to zero. Thus, TPE does not reveal itself (it becomes unobservable) in the crystal free from any external mechanical action under universally accepted measurement conditions, when electrodes completely cover the lateral opposite faces of the plate, normal to the spontaneous polarization vector. In spite of the fact that the TPE shares a common nature with the secondary pyroelectric effect, it differs fundamentally from both the primary and secondary pyroelectric effects by its symmetry [7] resulting in TPE existence in the crystals not belonging to the polar classes, which essentially extends the class of crystals used as sensitive elements in pyroelectric IR sensors. The system of signal electrodes for the information processing from a sensitive element of TPE sensor owing to the features in symmetry properties of this effect is of a radically different kind from those electrodes used in the classical primary-pyroeffect sensors.

The watt-joulemeter sensitive element being a rectangular thin crystal plate of the thickness $\ell$ free of any external mechanical action is subjected to a spatially inhomogeneous heating. Peculiarities of induced polar states in the crystal are determined by a character of this inhomogeneous temperature field. We shall consider such heating when the temperature varies only along the crystal plate depth. This distribution takes place under uniform irradiation of the front plate face by an incident sine-modulated radiation flux with a wavelength inside the strong lattice absorption band of the crystal. The plate, being a specific crystallographic cut of the used crystal, is made in such a manner that the tertiary polarization component is generated in the plate plane under the nonequilibrium thermal conditions considered. It should be pointed out that transverse TPE (when the polarization vector perpendicular to the radiation flux lies in the plane of the plate) may occur in the nonpolar cuts too. The polar state in the plate was proved to be immediately connected with a variation of its shape resulting from inhomogeneous heating the plate and generation of the flexural vibrations in it [8]. The plate volume is partitioned into the three parallel layers. The direction of the polarization vector $\vec{P}$ and sign of the components $\sigma_{i k}$ are constant in each of these layers each. Such a partition of the plate volume provides a minimum of its elastic energy during the shape variation resulting from inhomogeneous heating the plate. The tertiary polarization component is oppositely directed in adjacent layers, and its mean value in the plate is equal to zero. Neutral surface, on which the thermoelastic stresses and consequently polarization are unavailable, divides the plate thickness into two parts in the ratio 2:1 (an abrupt change of a sign of the tensor components of thermoelastic stress in a near-surface crystal region is neglected).

The revealed peculiarities in the polarization distribution over the plate thickness make it possible developing a radically new multifunctional pyroelectric device with two measuring channels and original system of electrically separated electrodes corresponding to this spatial distribution. The distinctive feature of their construction - the set of electrodes has to be deposited in such a way that the polarization charges are to collect from the unipolar parts of the lateral crystal surface, in which the potentials should be of the same sign. Two electrically insulated from one another electrodes in a form of metallic strips, covering the sections of a surface with the bound charges that are identical in sign, have to be deposited on the lateral plate surface perpendicular to the polarization vector. Continuous common electrode is deposited on the opposite lateral face. Each of electrodes must be connected to its own matching cascade, creating the corresponding channel of the measurement. Electrode adjacent to the irradiated surface is connected to the measuring circuit with a low load resistance. The

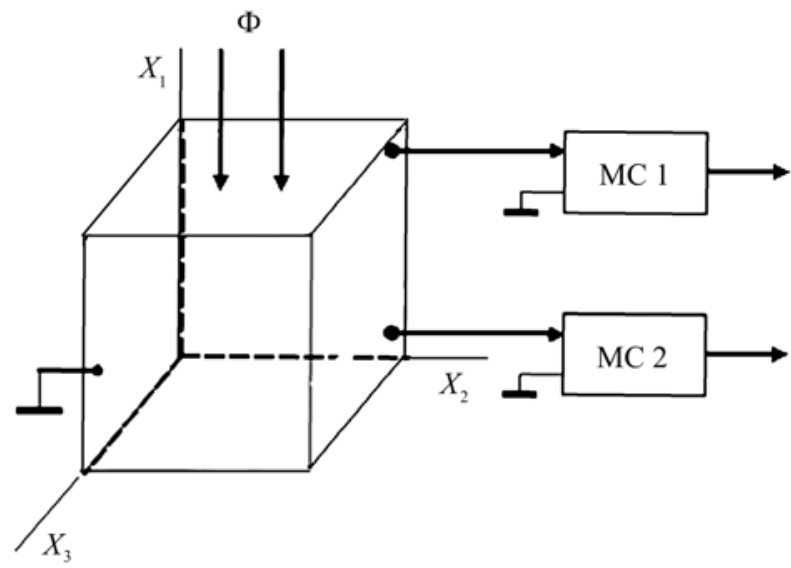

Fig. 1. Schematic arrangement of the separated electrodes for a sensitive element of the multifunctional pyroelectric sensor. $\mathrm{MC}$ - matching cascade for power measuring channel (1) and energy (2). 
electric signal picked off the heated part will be proportional to a power of the radiation pulse, producing a power measuring channel by this means. Another electrode is loaded on the matching cascade with a high input resistance with the aim of forming the energy measuring channel.

Watt-joulemeter under consideration has been developed on $\mathrm{LiNbO}_{3}$ crystal with the use of its different crystallographic cuts. This crystal is a traditional material for pyroelectric applications and its properties have been studied comprehensively in inhomogeneous temperature fields [9-10]. Sensitive element of the wattjoulemeter is the $X_{1}$-cut of the crystal (the cut plane is perpendicular to the crystallophysical $X_{1}$ axis), whereas the electrodes are deposited on the $X_{2}$ face. Indicated configuration of a sensitive element will be denoted the $X_{1} X_{2}$ configuration. A possibility of a variation in question for the sensor sensitive element is evident from the symmetry considerations [11] according to which, under inhomogeneous heating along the $X_{1}$ axis among all the symmetry elements of $\mathrm{LiNbO}_{3}$ crystal, only the symmetry plane perpendicular to this axis is retained. The sole polar direction (regarding the tertiary polarization component) induced in this plane is not coincided with the sole polar direction of the unperturbed crystal. The component of the tertiary polarization vector along the $X_{2}$ axis makes the needed property available for a sensor operating.

Amplitude-frequency and phase-frequency characterristics for the power measuring channel (the first channel) and energy one (the second channel) are represented in Figs 2 and 3, respectively. The time constant of the first channel derived from a behavior evaluation of its frequency characteristic at high modulation frequencies accounts for $10^{-4} \mathrm{~s}$. The time constant of the second channel was determined in response to the action in the form of step and was equal to $0.3 \mathrm{~s}$. This value is less than the electric time constant

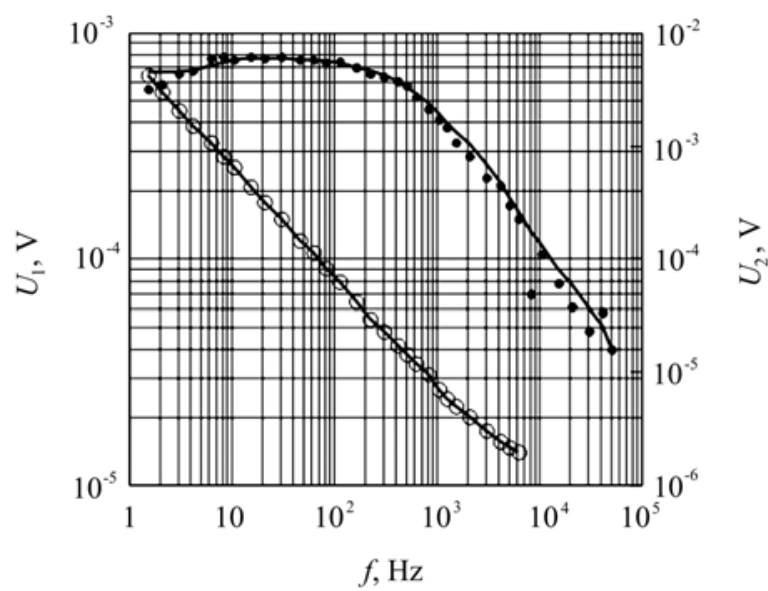

Fig. 2. Frequency dependence of the output signal amplitude for the watt-joulemeter. Solid symbols correspond to power measuring channel, while open ones to energy measuring channel.

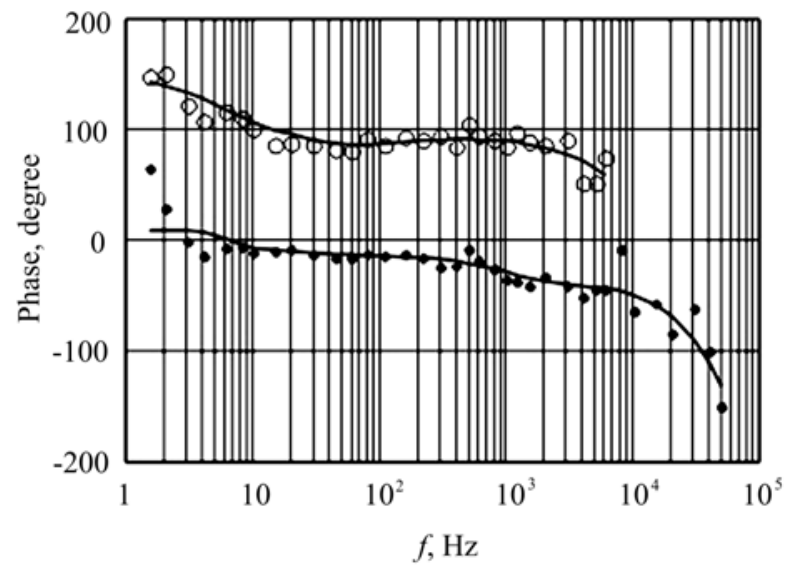

Fig. 3. Phase-frequency characteristics for measuring channels of power $(\bullet \bullet \bullet)$ and energy (ooo).

$\tau_{e}$ of a matching cascade by the factor of 3 . The reason of this discrepancy will be established later. As illustrated in Fig. 3, the phases of signals in both channels at all modulation frequencies differ by $\pi / 2$ according to the theoretical considerations.

Watt-joulemeter can also be designed in the $X_{1} X_{3}$ configuration. In this instance, the sensor is used for measuring the middle powers and energies, when the temperature on irradiated pyroelement surface is deficient to manifest the nonlinear crystal properties under radiation registration. The output electric signal generated by the heated crystal region presents the measured signal contribution due to the primary and secondary pyroeffects together with the tertiary pyroelectric component, with the most its part - at the expense of the primary component. Because of this, to sufficient accuracy, the speed of response of the first channel is determined only by the electric time constant $\tau_{e}$ and can be done high. A small value of $\tau_{e}$ provides not only a high temporal channel resolution, but also a fast screening of a polar state in the heated crystal region, eliminating the unwanted electrostatic pickup from the first channel to the second one.

Linearity of the watt-joulemeter of the $X_{1} X_{2}$ configuration with reference to the pulse energies was tested over a wide range of their values. A high degree of linearity of the volt-joule sensitivity of the second channel over a range of the energy densities $\left(10^{-3}-1\right) \mathrm{J} / \mathrm{cm}^{2}$ was established. Such behavior of the transformation coefficient well correlates with the theoretical examinations when the value of the tertiary polarization component is expressed in terms of the piezomodulus $d_{22}$. Among all the piezomoduli of the crystal under investigation, this is the most temperaturestable one. Typical values of the transformation coefficients over the energy and power measuring channels are $60 \mathrm{~V} / \mathrm{J}$ and $1 \mathrm{~V} / \mathrm{W}$, respectively. 


\section{Dynamic properties of the multifunctional device}

Ideal sensor is characterized by two time constants $\tau_{\text {th }}$ and $\tau_{e}[12]$. The first constant $\tau_{t h}$, being the thermal time constant, describes a time of the temperature establishment in a sensitive element in the process of a thermal interaction with the environment. This constant determines a low-frequency fall of the frequency sensor characteristic. Relaxation process of the electric crystal state under interaction with a measuring circuit is defined by the electric time constant $\tau_{e}$. This constant determines a high-frequency fall of the frequency sensor characteristic. Ideal pyroelectric sensor is the device operating only on the primary pyroelectric effect. The availability of macroscopic deformation in the crystal does not warrant the occurrence of this effect [13]. However, in actual practice, this deformation can not be obviated, and so the resonant properties of spatially limited media always manifest themselves. Frequency sensor characteristic contains all resonances, whereas the inertial properties of a real sensor are characterized by the relaxation times corresponding to resonant frequencies.

Consider the inertial properties of the device under study over the range of low frequencies. As is evident from the foregoing, the pulse energy measuring channel operates as an integrator of the bound charge generated in the cold crystal region. Integration will be incomplete, if the heat diffusion in a sensitive element has a strong impact on the temperature distribution during pulse time and hence causes a destruction of the polar state. In other words, the plate flexure tends to decrease with smoothing the temperature gradient in it. In this connection, the condition of registration of a radiation pulse with a duration $\tau$ is the fulfillment of the following inequality: $\tau<\tau_{d}=\ell^{2} / a$, where $a$ is the thermal diffusivity, $\tau_{d}$ is the diffusion time constant (it is suggested that $\tau_{t h}<\tau_{d}$ ). It is just this mechanism of a destruction of the polar state in the plate that is the reason of the discrepancy between the calculated and measured values of the time constant of the second channel as discussed in the preceding section. Notice that, for a destruction of the polar state, the total temperature equalization in the crystal is not required because the thermoelastic stresses, bringing about TPE, disappear even under any linear distribution of the temperature in a sample [14].

Inertial properties of the sensor under investigated over the range of high frequencies are determined by both the secondary and tertiary pyrocurrent resonances. These resonances, as well as pyroeffects, are difficult to separate, since they have the common piezoelectric nature. Nevertheless, taking into consideration the secondary pyroeffect symmetry, one can contend that if the resonance generates along direction perpendicular to the sole polar one ( $X_{1} X_{2}$ configuration), it corresponds to the resonance of the tertiary pyrocurrent component. What's more, the resonant frequencies of the pyrocurrents being considered have a different dependence on physical and geometric parameters of the crystal plate. Resonances of the tertiary pyrocurrent are determined by resonances of the flexural vibrations, which have been studied in detail in our paper [15]. The first resonance frequency of the flexural vibrations is as follows

$f_{r}=\frac{\mu_{k}^{2} \ell}{4 \pi R^{2}}\left(3 \rho S_{11} \beta_{1} \beta_{2}\right)^{-1 / 2}$,

where $R$ is the plate radius, $\rho$ is the material density, $\beta_{1,2}=1 \mp S_{12} / S_{11}, S_{11}$ and $S_{12}$ stand for the elastic compliance coefficients, $\mu_{k}$ are the solutions of a some transcendental equation (this equation is varied for the different boundary conditions). It should be pointed out that if the plate is illuminated uniformly, there are no flexural vibrations in its plane. Vibrations are not produced in the edge-clamped plate too. For the plate made of $\mathrm{LiNbO}_{3}(1 \mathrm{~mm}$ in thickness and $15 \mathrm{~mm}$ in radius), the numerical values of the fundamental resonant frequency are equal to 11.7 and $12.2 \mathrm{kHz}$ for the electrically free crystal and electrically clamped one, respectively.

Consider the contribution of the high-frequency resonances generated in the direction of the plate thickness to the inertial sensor properties. Occurrence of these resonances represents a response of external circuit to the reflections of the wave package, when repeated many times. This package is generated in the nearsurface plate region during its pulse heating. Since the wave package represents superposition of the plane waves, it needs to be ascertained what wave types are available in the plate under study. It turns out that three waves can propagate in the direction of the $X_{1}$ axis. Two waves are the transverse those with the polarization vectors that lie in the crystal symmetry plane, the third wave is longitudinal and travel along $X_{1}$. The longitudinal wave is an ordinary always, whereas the other waves are extraordinary and travel along $\vec{n} \| O X_{1}$ with the different velocities. From these considerations, in the general case there are three sets of the resonant frequencies for these waves. Only resonances associated with piezoactive vibrations will produce the electric response. The wave is piezoactive only when the following condition is fulfilled [14]

$$
\vec{g} \vec{q} \neq 0 \text {. }
$$

Here $\vec{q}$ is the polarization vector of a wave, the vector $\vec{g}$ has the following components $g_{k}=e_{i j k} n_{i} n_{j}, e_{i j k}$ are the piezoelectric coefficients, $n_{i}$ are the components of the unit vector normal to the plate. According to the Curie principle, symmetry of the vector $\vec{g}$ is described by the group $G(\vec{g})$ determined by the following relationship

$G(\vec{g}) \supseteq G\left(e_{i j k}\right) \cap G(\vec{n} \vec{n})$. 


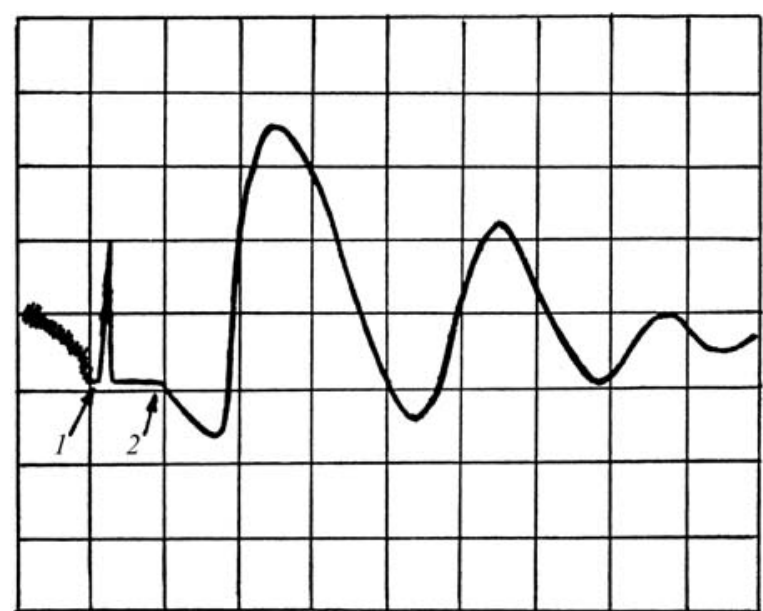

Fig. 4. Oscillogram of the electric signal generated in a twolayer system "dielectric-ferroelectric". Scale on the ordinate axis $-0.2 \mathrm{~V} / \mathrm{div}$, on the abscissa $-0.5 \mu \mathrm{s} / \mathrm{div}$.

In (4) $G\left(e_{i j k}\right)$ and $G(\vec{n} \vec{n})$ are the symmetry groups of the tensor $e_{i j k}$ and the dyad tensor $(\vec{n} \vec{n})$.

Analysis of the relationship (3) reveals that the vector $\vec{g}$ lies in the crystal symmetry plane and only the transverse waves are piezoactive. It is of interest that piezoresonances caused by these waves are not available in the external circuit. This is directly related to the construction of the sensitive element in which the electric field intensity induced by the resonances is parallel to the electrode plane on the lateral plate faces. Taking into account that the longitudinal wave is not piezoactive, it may be concluded that there is no a contribution of the "thickness" resonances to inertial properties of the device. This conclusion is in contradiction with the experimental results. The sensitive element was made in the form of a two-layer system "dielectric-ferroelectric" with the aim of detailed investigation of the indicated conflict. The glass plate with $2-\mathrm{mm}$ thickness and the strong radiation absorption at the wavelength of $\mathrm{CO}_{2}$ laser was used as a dielectric layer. Another plate (1 mm in thickness) with a spontaneous polarization vector in its plane was made of $\mathrm{LiNbO}_{3}$ in the form of the $X_{1} X_{3}$ configuration. This system was irradiated by $\mathrm{CO}_{2}$ laser pulse with the duration $150 \mathrm{~ns}$. Mechanism of the piezoelectric signal generation is identical to above-described one. The oscillogram of the output signal is presented in Fig. 4. The start of irradiation (a signal from the reference transducer) and the start of the response from the sample under study are marked off by symbols " 1 " and " 2 ". Delay time of the signal is equal to $0.4 \mu \mathrm{s}$ as seen in Fig. 4. The time necessary to a sound wave to propagate up to the distance equal to the dielectric layer thickness coincides with the indicated delay. In the case under study, a response emergence is due to the longitudinal wave that seemingly is inconsistent with Eq. (2) that is true for unbounded medium. However, this wave generates a bound charge on the lateral surface of a restricted plate with the dimensions in its plane $0.5 \times 0.5 \mathrm{~cm}$. Really, a direct calculation of the electric polarization induced by this wave shows that its vector lies in the wave package plane and therefore gives rise to a response in external circuit.

Being based on the performed investigation, it may be deduced that inertial properties of the developed device are determined by the least frequency of the fundamental resonance calculated from the relationship (2) (for $\mu_{1}=3.106$ ). In addition, the following requirement must be satisfied - the integer numbers of half-wavelengths must be along the plate thickness $\ell$. The fundamental resonance frequency is $f_{L}=V_{L} / \ell\left(V_{L}\right.$ is the velocity of the longitudinal wave).

\section{Conclusions}

Expansion of pyroactive crystallographic classes in number through the use of spatially inhomogeneous heating the crystal is of interest to practical purposes. A novel concept advanced by authors on creating the unconventional pyroactive materials on the basis of existing both polar and nonpolar structures substantially enriches the pyroelectric materials science and engineering. Performed theoretical and experimental investigations of the induced polar states in crystals under nonequilibrium thermal conditions reveal the possibilities for developing the multifunctional receiving devices on the basis of the tertiary pyroelectric effect. Design principle of the watt-joulemeter for a simultaneous measurement of the power and energy of radiation pulses is proposed and realized.

\section{Acknowledgments}

This work was partly supported by the Science and Education Ministry of Ukraine under Project No. 6.2.7B (50-2003).

\section{References}

1. V.F. Kosorotov, L.S. Kremenchugskij, V.B. Samoilov, and L.V.Shchedrina, Pyroelectric effect and its practical applications, Naukova Dumka, Kiev (1989) (in Russian).

2. V.F. Kosorotov, L.S. Kremenchugskij, L.V. Shchedrina, Tertiary pyroelectric effect and its application for a registration of the pulse radiation, in: Production and application of ferro- and piezomaterials, p. 71-76, Moscow. Dom NauchnoTechn. Propagandy, Moscow (1984) (in Russian).

3. I.V. Blonsky, V.F. Kosorotov, L.V. Shchedrina, L.V. Levash, New pyroactive structures for infrared optoelectronics // SPIE Proc. 3890 , p. 55-62 (1999).

4. W. Cady, Piezoelectricity, McGraw-Hill Book Com., Inc New York, London (1946).

5. J.F. Nye, Physical properties of crystals. Their representation by tensors and matrices. Oxford University Press, London (1957). 
6. J.A. Cooper, A fast-response pyroelectric thermal detector // J. Sci. Instrum. 39, p. 467-472 (1962).

7. V.F. Kosorotov, L.V. Shchedrina, Symmetry of induced polar states in noncentral crystals under inhomogeneous heating // Condensed Matter Phys. 3, p. 827-833 (2000).

8. V.F. Kosorotov, L.S. Kremenchugskij, L.V. Shchedrina, L.V. Levash, Tertiary pyroelectric effect in lithium niobate and lithium tantalate crystals // Ferroelectrics 70, p. 27-37 (1986).

9. I.B. Schein, P.J. Cressman, L.E. Cross, Electrostatic measurement of tertiary pyroelectricity in partially clamped $\mathrm{LiNbO}_{3} / /$ Ibid. 22, p. 945-948 (1979).

10. I.B. Schein, P.J. Cressman, L.E. Cross, Electrostatic measurements of unusually large secondary pyroelectricity in partially clamped $\mathrm{LiNbO}_{3} / /$ Ibid. 22, p. 937-943 (1979).
11. V.F. Kosorotov, Symmetry properties of tertiary pyroelectric effect in inhomogeneous one-dimensional temperature fields // Neorganicheskie Materialy 31, p. 827-830 (1995) (in Russian).

12. L.S. Kremenchugskij, Ferroelectric radiation detectors, Naukova Dumka, Kiev (1989) (in Russian).

13. A.K. Tagantsev, Pyro-, piezo-, flexoelectric and thermopolarization effects in ionic crystals // Usp. Fiz. Nauk 152, p. 423-448 (1987) (in Russian).

14. Yu.P. Sirotin, M.P. Shaskolskaya, Fundamentals of crystallophysics, Nauka, Moscow (1975) (in Russian).

15. V.F. Kosorotov, L.S. Kremenchugskij, L.V. Levash, L.V. Shchedrina, Dynamic tertiary pyroelectric effect and its inertial properties // Ferroelectrics 160, p. 125-136 (1994). 